\title{
KRLS based EKF method for Commercial Aero-engine Onboard Adaptive Model and Health parameters Estimation
}

\author{
Jiahui $\mathrm{Gu}^{\mathrm{a}}$, Jinquan Huang ${ }^{\mathrm{b},{ }^{*}}$ and Feng $\mathrm{Lu}^{\mathrm{c}}$ \\ College of Energy \& Power Engineering, Nanjing University of Aeronautics and Astronautics, \\ Nanjing, Jiangsu, China \\ agujiahui419@126.com, bjhuang@nuaa.edu.cn, lufengnuaa@126.com \\ ${ }^{*}$ Corresponding author
}

Keywords: Aero-engine; Health Parameters; Kalman Filter; Kernel Recursive Least Squares

\begin{abstract}
Due to the fact that computational cost of aircraft engine component level model (CLM) is heavy while memory of engine monitoring unit (EMU) is limited, a machine learning algorithm: exponential weighted - sliding window - kernel recursive least squares (EW-SW-KRLS) algorithm is proposed to replace CLM for onboard application. The exponential weight character guarantees its tracking ability and the sliding window structure fixs its onboard memory budget so the KRLS based EKF method can be applied to track engine output measurements and estimate variations of engine components efficiencies and mass flow capacities which are referred to as health parameters. Several kinds of performance degradations of a commercial aircraft turbofan engine have been numerically simulated and both the combined method and the traditional CLM based EKF method are applied to. The simulation results reveal that the proposed method is close to the CLM based EKF method in term of estimation accuracy with much less computation time and capable of onboard using.
\end{abstract}

\section{基于核递推最小二乘的扩展卡尔曼滤波算法的商用航空发动机 机载自适应模型与健康参数估计}

\author{
顾嘉辉 ${ }^{a}$ ，黄金泉 ${ }^{b}{ }^{*}$ ，鲁 峰 ${ }^{c}$
}

南京航空航天大学能源与动力学院, 南京, 江苏, 中国

agujiahui419@126.com, bjhuang@nuaa.edu.cn, lufengnuaa@126.com

*通讯作者

关键词：航空发动机；健康参数；卡尔曼滤波；核递推最小二乘

中文摘要. 针对航空发动机部件级模型 (CLM) 计算量大而机载EMU内存有限, 提出一种利 用机器学习算法：指数加权-滑动窗口-核递推最小二乘（EW-SW-KRLS）算法来代替部件级 模型机载使用的方法。其指数加权的特性保证了跟踪能力而滑动窗口结构维持了固定的机载 内存开销使得基于该核递推最小二乘的扩展卡尔曼滤波（EKF）算法可以用来跟踪发动机可 测输出同时估计发动机各部件效率和流量的变化量, 即健康参数。数字仿真模拟了某型商用 浴扇发动机的几种性能退化情况，同时采用该方法与传统的基于CLM的EKF方法进行比较。 经验证，该方法相比基于CLM的EKF方法在估计精度上非常接近，同时具有较少的计算时间， 适合机载使用。 


\section{1. 引言}

航空发动机由于长期工作在高温、高压、高转速的恶劣条件下，会发生不可避免的性能 退化。研究人员通过收集航空发动机的机载传感器数据, 对发动机的状态进行监视、跟踪与

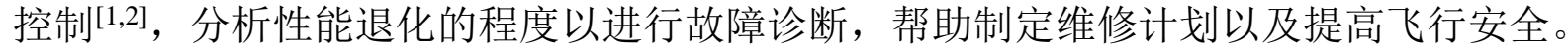

航空发动机的机载自适应模型是实现以上功能的基础。其中，机载模型的性能状态常用 各部件的流量和效率的相对变化量 (即健康参数) 来表示。由于健康参数无法直接测量, 只 能根据机载传感器的测量值, 如转子转速和各个截面的压力和温度通过一些滤波算法进行估 计与跟踪。然而, 航空发动机非线性特性很强, 现阶段的基于线性卡尔曼滤波算法的机载自 适应模型能实现一定跟踪效果, 但是由于线性模型精度较低, 健康参数的估计精度会随着飞 行循环数的增加而增加 ${ }^{[3]}$ ，而基于部件级模型（CLM）的非线性卡尔曼滤波算法 ${ }^{[4]}$ ，如扩展 卡尔曼滤波 (EKF) 算法虽然精度较高, 但是部件级模型需要迭代计算, 其计算量较大, 因 此目前机载应用仍以线性模型为主。

针对以上问题, 本文结合机器学习和扩展卡尔曼滤波算法, 通过核递推最小二乘算法学 习发动机状态 (包含健康参数) 与机载传感器输出之间的非线性关系, 然后利用该模型代替 航空发动机部件级模型, 实现基于扩展卡尔曼滤波算法的自适应跟踪与健康参数估计, 达到 对发动机性能状态的实时跟踪的目的。

\section{2. 指数加权-滑动窗口-核递推最小二乘算法}

最小二乘算法是最优状态估计中基础的一个方法，也是核递推最小二乘算法的基础。假 设一个含测量噪声的系统为:

$$
\boldsymbol{y}=\boldsymbol{H} \boldsymbol{x}+\boldsymbol{v}
$$

式中, $\boldsymbol{y}$ 为观测向量, $\boldsymbol{x}$ 为输入向量, $\boldsymbol{H}$ 为系数矩阵, $\boldsymbol{v}$ 为测量噪声。向量 $\boldsymbol{x}$ 的最可能值 满足如下目标函数最小:

$$
J=(\boldsymbol{y}-\boldsymbol{H} \hat{\boldsymbol{x}})^{T}(\boldsymbol{y}-\boldsymbol{H} \hat{\boldsymbol{x}})
$$

对 $J$ 求导可得 $\boldsymbol{x}$ 的估计值 $\hat{\boldsymbol{x}}$ :

$$
\hat{\boldsymbol{x}}=\left(\boldsymbol{H}^{T} \boldsymbol{H}\right)^{-1} \boldsymbol{H}^{T} \boldsymbol{y}
$$

核递推最小二乘算法 ${ }^{[5]}$ 是一种在特征空间通过 “核技巧” 利用最小二乘来拟合非线性映 射的算法。为了获得更好的泛化能力和预测精度, 该算法往往需要大量的训练样本, 但相应 会造成模型结构过于复杂、计算成本过高等问题。为了解决样本数量不断增加的问题, 有学 者提出了一些样本稀疏化的方法来精简模型的结构, 如近似线性依赖技术(Approximate Linear Dependency, ALD $)^{[5]}$ 以及滑动窗口 $(\text { Sliding-Window, SW })^{[6]}$ 等。而针对随时间动态变化系统的 跟踪问题, 有采用带遗忘因子的指数加权方法(Exponential weighted, EW $)^{[5]}$ 来提高该算法的跟 踪能力。

本文将指数加权与滑动窗口两种方法结合, 以期望同时达到跟踪性能与精简结构的目标。 指数加权-滑动窗口-核递推最小二乘算法(EW-SW-KRLS)结合如下:

设第 $i$ 步的输入向量为 $\boldsymbol{u}(i)$ 变换到特征空间 $\boldsymbol{F}$ 后记为 $\boldsymbol{\varphi}(i)$, 对应的输出观测值（标量）为 $d(i)$ 。设核函数为 $\kappa(\cdot, \cdot)$, 正则化参数为 $\lambda$, 滑动窗口大小为 $M$, 遗忘因子为 $\beta$ 。完成以下步骤:

1. 初始化: 第 $i=1$ 步, 有:

$$
\begin{aligned}
& Q(1)=(\lambda \beta+\kappa(\boldsymbol{u}(1), \boldsymbol{u}(1)))^{-1} \\
& a(1)=Q(1) d(1)
\end{aligned}
$$

初始字典容量为 1 , 记作 $d i c_{-}$num $=1$ 。 
2. $i=i+1$, 更新第 $i$ 步的 $\boldsymbol{Q}(i)$ 与 $\boldsymbol{a}(i)$ :

若 $d i c_{-}$num $\leq M$, 则字典继续增加样本:

$$
\begin{aligned}
\boldsymbol{h}(i) & =\left[\begin{array}{lll}
\kappa(\boldsymbol{u}(i), \boldsymbol{u}(1)) & \ldots & \kappa(\boldsymbol{u}(i), \boldsymbol{u}(i-1))
\end{array}\right]^{T} \\
\boldsymbol{z}(i) & =\boldsymbol{Q}(i-1) \boldsymbol{h}(i) \\
r(i) & =\lambda \beta^{i}+\kappa(\boldsymbol{u}(i), \boldsymbol{u}(i))-z^{T}(i) \boldsymbol{h}(i) \\
\boldsymbol{Q}(i) & =r^{-1}(i)\left[\begin{array}{cc}
\boldsymbol{Q}(i-1) r(i)+z(i) \boldsymbol{z}^{T}(i) & -z(i)
\end{array}\right] \\
\boldsymbol{d}(i) & =\left[\begin{array}{cc}
\boldsymbol{d}^{T}(i-1) & d(i)
\end{array}\right]^{T} \\
\boldsymbol{a}(i) & =\boldsymbol{Q}(i) \boldsymbol{d}(i)
\end{aligned}
$$

同时 $d i c_{-} n u m=d i c_{-} n u m+1$ 。若 $d i c_{-} n u m=M+1$, 定义: $\boldsymbol{A}_{(i: j, m: n)}$ 为取 $\boldsymbol{A}$ 矩阵的第 $i$ 行到第 $j$ 行, 第 $m$ 列到第 $n$ 列的所有元素构成的新矩阵，则删去当前字典中的第一项:

$$
\begin{aligned}
& e=\boldsymbol{Q}(i-1)_{(1,1)} \\
& \boldsymbol{F}=\boldsymbol{Q}(i-1)_{(2: M, 1)} \\
& \boldsymbol{G}=\boldsymbol{Q}(i-1)_{(2: M, 2: M)} \\
& \boldsymbol{Q}_{d s}=\boldsymbol{G}-\boldsymbol{F} \boldsymbol{F}^{T} / e \\
& \boldsymbol{h}(i)=\left[\begin{array}{lll}
\kappa(\boldsymbol{u}(i), \boldsymbol{u}(i-M+1)) & \ldots & \kappa(\boldsymbol{u}(i), \boldsymbol{u}(i-1))
\end{array}\right]^{T} \\
& \boldsymbol{z}(i)=\boldsymbol{Q}_{d s} \boldsymbol{h}(i) \\
& r(i)=\lambda \beta^{i}+\kappa(\boldsymbol{u}(i), \boldsymbol{u}(i))-\boldsymbol{z}^{T}(i) \boldsymbol{h}(i) \\
& \boldsymbol{Q}(i)=r^{-1}(i)\left[\begin{array}{ll}
\boldsymbol{Q}_{d s} r(i)+\boldsymbol{z}(i) \boldsymbol{z}^{T}(i) & -\boldsymbol{z}(i)
\end{array}\right] \\
& \boldsymbol{d}(i)=\left[\begin{array}{ll}
\boldsymbol{d}^{T}(i-1)_{(2: M, 1)} & d(i)
\end{array}\right]^{T} \\
& \boldsymbol{a}(i)=\boldsymbol{Q}(i) \boldsymbol{d}(i)
\end{aligned}
$$

3. 然后利用该模型预测第 $i+1$ 步的输出:

$$
\begin{aligned}
& \hat{d}(i+1)=\boldsymbol{\varphi}^{T}(i+1)[\boldsymbol{\varphi}(i-M+1) \quad \ldots \quad \boldsymbol{\varphi}(i)] \boldsymbol{a}(i) \\
& =\sum_{j=i-M+1}^{i} a_{j}(i) \kappa(\boldsymbol{u}(i+1), \boldsymbol{u}(j))
\end{aligned}
$$

如果当前步数小于总步数，则返回 2 ，否则结束。

由式(4)到式(7)可见, 该算法的计算全部为矩阵计算, 且矩阵的维数增加到滑动窗口大小 $M$ 后不再增加, 故每次的计算量较小且稳定。

\section{3. 数字仿真验证}

本节研究利用核递推最小二乘算法代替发动机部件级模型, 实现基于扩展卡尔曼滤波算 法的某型商用航空发动机机载自适应跟踪与健康参数估计。

\section{1 某型商用航空发动机介绍}

本文代替真实发动机进行数字仿真的模型为某型大涵道比浴扇发动机部件级模型, 如图1 所示, 共有 5 个旋转部件: 风扇、低压压气机、高压压气机、高压浴轮和低压涡轮。关于部件 级模型建模方面的内容, 可参考文献[7]。 


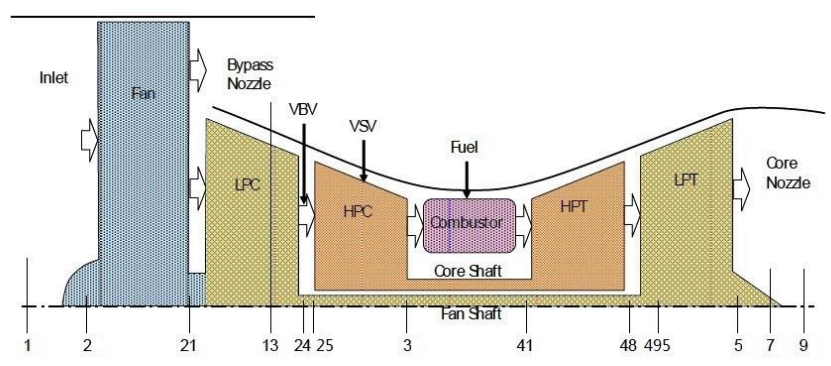

图1 某型大涵道比浴轮风扇发动机图及截面编号

健康参数为各个部件效率/流量的相对变化量:

$$
\left\{\begin{array}{l}
S E_{i}=\frac{\eta_{i}}{\eta_{i}^{*}}-1 \\
S W_{i}=\frac{W_{i}}{W_{i}^{*}}-1
\end{array} i=1,2,3,4,5\right.
$$

式中, $S E$ 与 $S W$ 分别表示效率与流量健康参数, $\eta_{i}$ 和 $W_{i}$ 分别为部件效率和流量的实际值, $\eta_{i}^{*}$ 和 $W_{i}^{*}$ 分别为部件没有发生性能退化, 即设计状态时的效率和流量, 下标 1 至 5 分别对应 5 个部 件。初始时刻假设无任何性能退化, 所有健康参数为 0 。与气路相关的可测参数如表 1 所示, 并假设机载传感器含高斯白噪声, 满足 $\boldsymbol{v}_{k} \sim N\left(\boldsymbol{0}, \boldsymbol{R}_{k}\right)$, 其中 $\boldsymbol{R}_{k}=0.002^{2} \boldsymbol{I}_{9 \times 9}$ 。

\begin{tabular}{|c|c|c|}
\multicolumn{2}{c|}{ 表 1 可测参数 } \\
\hline 序号 & 符号 & 可测参数名称 \\
\hline 1 & $N_{1}$ & 低压转子转速 \\
\hline 2 & $N_{2}$ & 高压转子转速 \\
\hline 3 & $T_{13}$ & 风扇出口总温 \\
\hline 4 & $P_{13}$ & 风扇出口总压 \\
\hline 5 & $T_{25}$ & 高压压气机进口总温 \\
\hline 6 & $P_{25}$ & 高压压气机进口总压 \\
\hline 7 & $T_{3}$ & 高压压气机出口总温 \\
\hline 8 & $P_{3}$ & 高压压气机出口总压 \\
\hline 9 & $T_{495}$ & 排气温度 \\
\hline
\end{tabular}

\section{2 核递推最小二乘的扩展卡尔曼滤波算法初始化}

关于EKF算法可以参考文献[8]，本文利用 $E K F$ 实现自适应跟踪与健康参数估计。考虑到 可测参数仅有 9 个以及卡尔曼滤波算法中状态量的可观性要求, 选取待估计的健康参数为不包 含高压涡轮流量和低压涡轮流量的 8 个健康参数。

将第2节介绍的EW-SW-KRLS算法代替部件级模型作为机载模型使用。选取核函数为高 斯核:

$$
\kappa(\boldsymbol{x}, \boldsymbol{y})=\exp \left(-\frac{\|\boldsymbol{x}-\boldsymbol{y}\|^{2}}{2 \sigma^{2}}\right)
$$

其中, 核参数: $\sigma=2$, 正则化参数: $\lambda=0.01$, 设滑动窗口大小: $M=100$ 。输入样本选取 为该发动机的状态量: 包含发动机运行状态的两个转子转速以及 8 个健康参数:

$$
\boldsymbol{x}=\left[\begin{array}{llllllllll}
N_{1} & N_{2} & S W_{1} & S E_{1} & S W_{2} & S E_{2} & S W_{3} & S E_{3} & S E_{4} & S E_{5}
\end{array}\right]^{T}
$$

由于输出样本是 9 个可测输出, 所以分别建立 9 个KRLS模型。从式(6)可知, 9个KRLS模 型的 $\boldsymbol{Q}(i)$ 矩阵相同, 无需重复计算, 唯一不同点仅在最后一步计算 $\boldsymbol{a}(i)$ 上, 所以 9 个KRLS模型 的计算量相比1个KRLS模型只是略有增加。 
由于初始时, KRLS模型中没有任何样本, 故取前100次飞行数据及其对应的基于部件级 模型估计得到的健康参数值作为KRLS模型的初始样本, 构成初始字典。从第101次飞行开始, 每隔35个飞行循环，离线或在地面基站 ${ }^{[9]}$ 用基于CLM的EKF算法估计出当前发动机的性能退 化情况, 然后将当前估计出的健康参数值与对应的飞行数据增加到机载KRLS模型里以保证模 型的精度，同时删去距离当前飞行循环最久的一组样本，保持模型的窗口大小不变。

\section{3 机载自适应跟踪与健康参数估计}

本文的数字仿真是在台式机上的MatLab R2012a中运行, 该台式机的配置如下：32位XP 操作系统、Core i5-650双核CPU@3.20GHz, 1.18GHz以及2.99GB的内存。

在该商用航空发动机的经济巡航点, 模拟稳定状态下性能缓慢退化的过程。数字仿真的 采样周期为 $25 \mathrm{~ms}$, 一个周期对应一个飞行循环, 共 4500 个采样周期。性能退化数据参考NASA 的Glenn研究中心在MAPSS(Modular Aero Propulsion System Simulation)仿真平台中浴扇发动 机完成一定工作循环数后气路健康参数变化情况的统计数据 ${ }^{[10]}$, 共设置4种性能退化情况, 其 中2种包含气路突变故障且假设都发生在第2102个飞行循环时）。

表 24500 飞行循环数后的气路健康参数变化情况（\%)

\begin{tabular}{|c|c|c|c|c|c|c|c|c|c|}
\hline \multirow{2}{*}{ 数字仿真序号 } & \multicolumn{2}{|c|}{ 风扇 } & \multicolumn{2}{|c|}{ 低压压气机 } & \multicolumn{2}{|c|}{ 高压压气机 } & \multirow{2}{*}{$\begin{array}{c}\text { 高压涡轮 } \\
\text { 效率 }\end{array}$} & \multirow{2}{*}{$\frac{\text { 低压浴轮 }}{\text { 效率 }}$} & \multirow{2}{*}{ 变化形式 } \\
\hline & 流量 & 效率 & 流量 & 效率 & 流量 & 效率 & & & \\
\hline 1 & -3 & -2.5 & -3.5 & -2 & -9 & -6 & -4 & -1 & 线性 \\
\hline 2 & -1 & -5 & -2 & -4 & $\begin{array}{c}-5.5+ \\
(-1) \\
\end{array}$ & $\begin{array}{l}-4.5+ \\
(-1.5) \\
\end{array}$ & -2.5 & -2 & 线性+突变故障 \\
\hline 3 & -4 & -1.5 & -2 & -3 & -6 & -8 & -2.5 & -3 & $a k^{3}+b$ \\
\hline 4 & $\begin{array}{c}-2+ \\
(-1.5)\end{array}$ & $\begin{array}{l}-4+ \\
(-2)\end{array}$ & -1 & -2.5 & -8 & -7 & -4.5 & -0.5 & $a e^{b k}+$ 突主 \\
\hline
\end{tabular}

限于篇幅, 仅给出数字仿真 2 的自适应模型跟踪排气温度 $T_{495}$ 的结果与健康参数估计结果, 如图2与图3。图2中后缀 obsv 表示传感器测量值, 后缀 $k r l s$ 表示KRLS模型估计值, 后缀 real 表 示添加噪声前数字仿真模型的输出值。

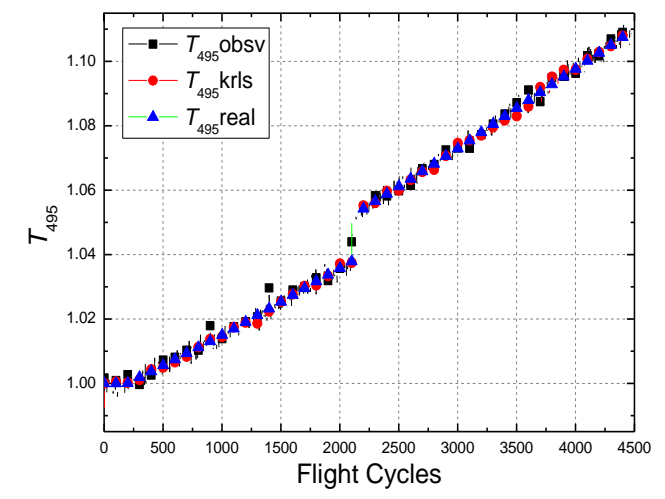

图2 基于KRLS的EKF算法的自适应模型跟踪排气温 度结果 (数字仿真2)

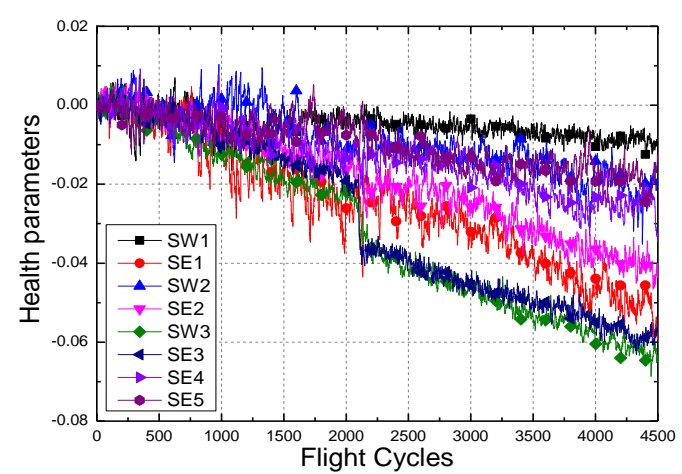

图3 基于KRLS的EKF算法的健康参数估计结果 (数字 仿真2)

将表 2 中所有数字仿真的结果以健康参数估计值的均方根误差总和 $(\operatorname{sum}(R M S E)$ 计算方法 如式(11))的形式总结于表3中。

$$
\operatorname{sum}(R M S E)=\sqrt{\frac{\sum_{k=1}^{k=4500}\left(S \hat{W}_{1, k}-S W_{1, k}\right)^{2}}{4500}}+\ldots+\sqrt{\frac{\sum_{k=1}^{k=4500}\left(S \hat{E}_{5, k}-S E_{5, k}\right)^{2}}{4500}}
$$


表 34 种性能退化情况的数字仿真结果

\begin{tabular}{|c|c|c|c|c|c|c|c|c|}
\hline \multirow{2}{*}{ 方法 } & \multicolumn{2}{|c|}{ 数字仿真 1 } & \multicolumn{2}{|c|}{ 数字仿真 2} & \multicolumn{2}{|c|}{ 数字仿真 3} & \multicolumn{2}{c|}{ 数字仿真 4} \\
\cline { 2 - 10 } & $\begin{array}{c}\text { 计算 } \\
\text { 时间/s }\end{array}$ & $\operatorname{sum}(R M S E)$ & $\begin{array}{c}\text { 计算 } \\
\text { 时间/s }\end{array}$ & $\operatorname{sum}(R M S E)$ & $\begin{array}{c}\text { 计算 } \\
\text { 时间/s }\end{array}$ & $\operatorname{sum}(R M S E)$ & $\begin{array}{c}\text { 计算 } \\
\text { 时间/s }\end{array}$ & $\operatorname{sum}(R M S E)$ \\
\hline KRLS-EKF & 2.2477 & 0.02194 & 2.2449 & 0.02360 & 2.4391 & 0.02143 & 2.3099 & 0.02237 \\
\hline CLM-EKF & 22.7725 & 0.01830 & 23.1368 & 0.01883 & 22.3041 & 0.01744 & 22.6288 & 0.01838 \\
\hline
\end{tabular}

由图2、图3与表3可知, 该算法可以代替部件级模型, 实现模型的自适应跟踪与健康参数 的估计且具有非常小的计算量，适合机载使用。

\section{4. 结束语}

本文提出了利用核递推最小二乘模型代替航空发动机部件级模型的方法，通过扩展卡尔 曼滤波算法实现了发动机自适应跟踪与健康参数估计，相比基于部件级模型的扩展卡尔曼滤 波算法, 该方法能在牺牲较少估计精度的情况下，极大地降低计算时间，且每步的计算量稳 定。

\section{致谢}

本文为国家自然科学基金（编号51276087）与国家自然科学青年基金（编号61304133） 的阶段性成果之一。

\section{References}

[1] J. S. Litt, D. L. Simon and S. Garg, A survey of intelligent control and health management technologies for aircraft propulsion systems, Journal of Aerospace Computing Information \& Communication, vol. 12, pp. 543-563, 2005.

[2] C. H. Jiang, Z. Y. Sun and X. Wang, Critical technologies for aero-engine prognostics and health management systems development, Journal of Aerospace Power, vol. 11, pp. 2589-2594, 2009.

[3] X. Liu and N. Xue, An improved hybrid Kalman filter design for aircraft engine based on a velocity-based LPV framework, Control and Decision Conference, pp. 1873-1878, 2016.

[4] T. Kobayashi and D. L. Simon, Integration of On-Line and Off-Line Diagnostic Algorithms for Aircraft Engine Health Management, Journal of Engineering for Gas Turbines \& Power, vol 4, pp. 649-659, 2007.

[5] Y. Engel, S. Mannor and R. Meir, The kernel recursive least-squares algorithm, Signal Processing IEEE Transactions, vol. 8, pp. 2275-2285, 2004.

[6] V. S. Van, J. Via and I. Santamaria, A Sliding-Window Kernel RLS Algorithm and Its Application to Nonlinear Channel Identification, International Conference on Acoustics, pp. 789-792, 2006.

[7] S. G. Zhang, Y. Q. Guo and J. Lu, Research on aircraft engine component-level models based on GasTurb/MATLAB, Journal of Aerospace Power, vol. 12, pp. 2850-2856, 2012.

[8] F. Lu, J. Q. Huang and Y. Q. Lv, Gas Path Health Monitoring for a Turbofan Engine Based on a Nonlinear Filtering Approach, Energies, vol. 13, pp. 492-513, 2013.

[9] D. L. Simon, An Integrated Architecture for On-Board Aircraft Engine Performance Trend Monitoring and Gas Path Fault Diagnostics, NASA/TM-2010-216358, 2010.

[10]J. S. Litt, K. I. Parker and S. Chatterjee, Adaptive gas turbine engine control for deterioration compensation due to aging, NASA-TM-2003-212607, 2003. 